\title{
ON THE GROWTH OF ANALYTIC FUNCTIONS*
}

\author{
BY \\ NORMAN LEVINSON†
}

1. Pólya, $\ddagger$ in a restricted case, and Bernstein, $\S$ under rather general conditions, have, to state their results roughly, proved that the rate of growth of an analytic function along a line can be determined by its growth along a suitable sequence of discrete points on the line. In proving his results Bernstein uses certain rather deep theorems from the theory of Dirichlet series and points out $\uparrow$ that as yet no proof of the results has been obtained using ordinary function theory.

Here we shall give a simple function-theoretic proof of a set of theorems which include those of Bernstein. We shall then refine our methods and obtain a set of new theorems which are remarkably precise. (See Theorem VII for example.)

We shall deal exclusively with functions which are analytic in a sector and of order 1.\| For use here we can define the Phragmén-Lindelöf function for a function $f(z)$ analytic in a sector $|\operatorname{am} z| \leqq \alpha$, as

$$
h(\theta)=\limsup _{r \rightarrow \infty} \frac{\log \left|f\left(r e^{i \theta}\right)\right|}{r}, \quad|\theta| \leqq \alpha .
$$

The following theorems are among those which will be proved.

THEOREM I.** Let $\phi(z)$ be analytic in some sector $|\mathrm{am} z| \leqq \alpha$. Let $h(\theta)$ defined as in (1.0) be its Phragmén-Lindelöf function and let $h(0)=a$. Suppose

$$
h(\theta) \leqq a \cos \theta+b|\sin \theta|, \quad|\theta| \leqq \alpha .
$$

Let $\left\{z_{n}\right\}$ be a sequence of complex numbers such that

$$
\lim _{n \rightarrow \infty} \frac{n}{z_{n}}=D,
$$

* Presented to the Society, September 10, 1937; received by the editors March 4, 1937.

† National Research Fellow.

‡ G. P6lya, Untersuchungen über Lizcken und Singularitäten von Potenzreihen, Mathematische Zeitschrift, vol. 29 (1924).

$\S$ V. Bernstein, Généralisation et Conséquences d'un théorème de Le Roy-Lindelöf, Bulletin des Sciences Mathématiques, vol. 52 (1928); and Séries de Dirichlet, Chapter IX, Paris, 1933.

IV V. Bernstein, Series de Dirichlet, loc. cit., pp. 229 and 249.

$\|$ Functions of any other finite order can be transformed to functions of order 1 by $w=z^{\rho}$.

${ }^{* *}$ In the case of real $\left\{z_{n}\right\}$ a proof of this theorem and a related gap theorem were given by the author in the Bulletin of the American Mathematical Society, vol. 42 (1936), p. 702. 
where $D$ is real, and such that for some $d>0$

$$
\left|z_{n}-z_{m}\right| \geqq|n-m| d \text {. }
$$

If

$$
\pi D>b,
$$

then

$$
\underset{n \rightarrow \infty}{\limsup } \frac{\log \left|\phi\left(z_{n}\right)\right|}{\left|z_{n}\right|}=\limsup _{r \rightarrow \infty} \frac{\log |\phi(r)|}{r} .
$$

In the case where the sequence $\left\{z_{n}\right\}$ is real, Theorem $I$ is due to Bernstein. ${ }^{*}$ We observe that (1.2) requires, if $z_{n}=r_{n} e^{i \theta_{n}}$, that $n / r_{n} \rightarrow D$ and that $\left|\theta_{n}\right| \rightarrow 0$ as $n \rightarrow \infty$. In other words all except a finite number of the $z_{n}$ will lie in any sector containing the real axis.

A special case of Theorem $I$ is the following:

THEOREM II. Let $\phi(z)$ be analytic and of exponential type $†$ in the half-plane $\mid$ am $z \mid \leqq \frac{1}{2} \pi$. If $L$ is defined by

$$
h\left(\frac{1}{2} \pi\right)+h\left(-\frac{1}{2} \pi\right)=2 \pi L,
$$

and if $\left\{z_{n}\right\}$ is a sequence satisfying (1.2) and (1.3), then

$$
D>L
$$

implies (1.5).

It is easy to see by considering $\sin \pi z$ at the points $z_{n}=n$ that (1.7) is critical, for in this case $D=L=1$ and it is clear that (1.5) is not true. Nevertheless we shall show that the condition (1.7) can be weakened considerably without disrupting the theorem. It is in weakening this condition that we obtain a new and very precise set of theorems.

The simplest of these theorems is

THEOREM VII. Let $\phi(z)$ be analytic and of exponential type in the sector $|\operatorname{am} z| \leqq \frac{1}{2} \pi$. Let

$$
\phi(i y)=O(1), \quad|y| \rightarrow \infty .
$$

Let $\left\{z_{n}\right\}$ be a sequence of density $\ddagger D \geqq 0$, such that $\left|\mathrm{am} z_{n}\right| \rightarrow 0$ as $n \rightarrow \infty$ and

* Loc. cit., Chapter IX. Likewise Theorem II stated below is due to Bernstein in the case where the $\left\{z_{n}\right\}$ are real.

† A function $f(z)$ is of exponential type in a sector, $|\mathrm{am} z| \leqq \alpha$, if $f(z)=O\left(e^{C|z|}\right),|\mathrm{am} z| \leqq \alpha$, for some constant $C$.

$\ddagger$ In case the density of $\left\{z_{n}\right\}$ is greater than zero, Theorem II gives Theorem VII at once. Thus this theorem is really of interest when $D=0$ in which case Theorem II cannot be applied. 
$\left|z_{n}-z_{m}\right| \geqq|n-m| d . A$ necessary and sufficient condition that (1.5) hold is that

$$
\sum_{1}^{\infty} \frac{1}{\left|z_{n}\right|}=\infty \text {. }
$$

Clearly Theorem VII is a much sharper result than that obtained by trying to apply Theorem II. For example, if $z_{n}=n \log (1+n)$, Theorem VII is applicable while Theorem II is not.

The condition $\phi(i y)=O(1)$ in Theorem VII can easily be replaced by

$$
\int_{-\infty}^{\infty} \frac{\log ^{+}|\phi(i y)|}{1+y^{2}} d y<\infty \text {. }
$$

Analogous results are true in Theorem V.I which follows and in related theorems.

Another of these theorems is

TheOREM VI. Let $\phi(z)$ be analytic and of exponential type in the half-plane $|\mathrm{am} z| \leqq \frac{1}{2} \pi$. Let

$$
\phi(i y)=O\left(e^{\pi L|y|}\right)
$$

and let $\left\{\lambda_{n}\right\}$ be an increasing sequence of positive numbers satisfying

$$
\lim _{n \rightarrow \infty} \frac{n}{\lambda_{n}}=D, \quad \lambda_{n+1}-\lambda_{n} \geqq d>0 .
$$

Let $\Lambda(u)$ be the number of $\lambda_{n}<u$. If

$$
\int_{1}^{\infty} \frac{\Lambda(u)-u L}{u^{2}} d u=\infty
$$

and if*

$$
\Lambda(u)>L u+C
$$

for some $C$, then

$$
\limsup _{n \rightarrow \infty} \frac{\log \left|\phi\left(\lambda_{n}\right)\right|}{\lambda_{n}}=\limsup _{x \rightarrow \infty} \frac{\log |\phi(x)|}{x} .
$$

Theorem VI goes much further than Theorem II in that it does not exclude the possibility of $D=L$.

We shall first give simple function-theoretic proofs of Theorems I, II, and related results which are due to Bernstein in case $\left\{z_{n}\right\}$ is real. In $\$ 3$ we

* This condition is by no means critical. It can for example be replaced by $\Lambda(u)>L u-u^{\alpha}-C$, $\alpha<1$. However condition (1.10) is the divergence condition (1.8) of Theorem VII, and, as in that theorem, it is easy to show that it is a best possible result. 
will turn to the proofs of our new results such as Theorem VI, VII, and related theorems.

2. Here we concern ourselves with Theorems I and II and certain of their extensions.

The following result of Phragmén and Lindelöf will be of basic importance:*

THEOREM A. Let $f(z)$ be an analytic function of $z=r e^{i \theta}$, regular in the region $R$ between two straight lines making an angle $\pi / \alpha$ at the origin, and on the lines themselves. Suppose $|f(z)| \leqq M$ on the lines and that as $r \rightarrow \infty, f(z)=O\left(e^{r \beta}\right)$ uniformly in $R$ for some $\beta<\alpha$. Then $f(z) \leqq M$ throughout $D$.

We also require

LEMMA 1. Let $\left\{z_{n}\right\}$ satisfy (1.2) and (1.3). If

$$
F(z)=\prod_{1}^{\infty}\left(1-\frac{z^{2}}{z_{n}^{2}}\right)
$$

then along the line am $z=\theta$

$$
\lim _{r \rightarrow \infty} \frac{\log \left|F\left(r e^{i \theta}\right)\right|}{r}=\pi D|\sin \theta|, \quad \theta \neq 0, \pi .
$$

Also

$$
\limsup _{|x| \rightarrow \infty} \frac{\log |F(x)|}{|x|}=0
$$

Moreover

$$
\frac{1}{F(z)}=O\left(e^{|z| \log |z|}\right), \quad\left|z \pm z_{n}\right| \geqq \frac{1}{2} d,
$$

and for any $\epsilon>0$

$$
\frac{1}{F^{\prime}\left(z_{n}\right)}=O\left(e^{e\left|z_{n}\right|}\right)
$$

For real $\left\{z_{n}\right\}$ all these results are well known. $\dagger$ (2.3) can be made much more precise but suffices for our purposes. The proof of this lemma is quite straightforward. Since it is very much the same as for real $\left\{z_{n}\right\}$ we omit it.

* See, e.g., Titchmarsh, Theory of functions, Oxford, 1932, p. 177.

† F. Carlson, Über Potenzreihen mit endlich vielen verschiedenen Koeffizienten, Mathematische Annalen, vol. 79 (1919), pp. 237-245, especially pp. 239-240. 
Proof of Theorem I. We observe that there is no loss of generality in taking $\alpha<\frac{1}{2} \pi$. Clearly if (1.5) does not hold there exists a $c$ such that

$$
\limsup _{n \rightarrow \infty} \frac{\log \left|\phi\left(z_{n}\right)\right|}{\left|z_{n}\right|}<c<a=\limsup _{x \rightarrow \infty} \frac{\log |\phi(x)|}{x} \text {. }
$$

It follows from (2.4) and (2.5) that

$$
g(z)=\sum_{1}^{\infty} \frac{\phi\left(z_{n}\right) e^{-c z_{n}}}{F^{\prime}\left(z_{n}\right)\left(z-z_{n}\right)} e^{c z} F(z)
$$

is an entire function. Since $g\left(z_{n}\right)=\phi\left(z_{n}\right)$ it follows that

$$
\psi(z)=\frac{\phi(z)-g(z)}{F(z)}
$$

is analytic for $|\mathrm{am} z| \leqq \alpha$. Using (1.1), (2.3), and (2.6), it follows that for $\left|z-z_{n}\right| \geqq \frac{1}{2} d$

$$
\psi(z)=O\left(e^{|z| \log |z|}\right) .
$$

But $\psi(z)$ is analytic and (2.8) being true on the circles $\left|z-z_{n}\right|=\frac{1}{2} d$ must be true inside, since a function analytic in a domain takes its maximum value on the boundary of the domain. Therefore (2.8) holds in the entire sector.

From (1.1), (2.1), (2.6), and (2.7)

$\psi\left(r e^{ \pm i \alpha}\right)=O(\exp [r(a \cos \alpha+b \sin \alpha-\pi D \sin \alpha+\epsilon)]+\exp [c r \cos \alpha]), \epsilon>0$.

Or setting $(\pi D-b) \tan \alpha=\gamma$ we have

(2.9) $\psi\left(r e^{ \pm i \alpha}\right)=O(\exp [r \cos \alpha(a-\gamma+\epsilon \sec \alpha)]+\exp [\operatorname{cr} \cos \alpha])$.

Since $\pi D>b, \gamma>0$. If we take $\epsilon<\frac{1}{2} \gamma \cos \alpha$, then (2.9) becomes

$$
\psi\left(r e^{ \pm i \alpha}\right)=O(\exp [p r \cos \alpha]), \quad p=\max \left(a-\frac{1}{2} \gamma, c\right) .
$$

In other words $\psi(z) e^{-p z}$ is bounded for am $z= \pm \alpha$. But by Theorem $A$, this and (2.8) implies that it is bounded in the entire sector $(-\alpha, \alpha)$. Thus in particular

$$
\psi(x)=O\left(e^{p x}\right), \quad p=\max \left(a-\frac{1}{2} \gamma, c\right) .
$$

But by (2.7), $\phi(x)=\psi(x) F(x)+g(x)$. When we use (2.2), (2.6), and (2.10), this gives

$$
\limsup _{x \rightarrow \infty} \frac{\log |\phi(x)|}{x} \leqq \max \left(a-\frac{1}{2} \gamma, c\right) .
$$

This contradicts the assumption that $h(0)=a$ and proves Theorem I. 
Proof of Theorem II. There is no loss in generality in assuming that $h\left(\frac{1}{2} \pi\right)=h\left(-\frac{1}{2} \pi\right)=\pi L$.

Let us set

$$
\limsup _{x \rightarrow \infty} \frac{\log |\phi(x)|}{x}=a .
$$

Then clearly when we apply Theorem $\mathrm{A}$ to $\phi(z) \exp [(\pi L+\epsilon) i z-(a+\epsilon) z]$, $\epsilon>0$, in the upper right quadrant, it follows at once from Theorem $A$ that here

$$
h(\theta) \leqq a \cos \theta+\pi L|\sin \theta| .
$$

Similarly this holds in the lower right quadrant. Theorem II now follows at once from Theorem I.

THEOREM III. Let $\Phi(z)$ be an analytic function in the right half-plane $|\operatorname{am} z| \leqq \frac{1}{2} \pi$ such that

(2.11) $\Phi\left(r e^{i \theta}\right)=O(\exp [(a \log r \cos \theta+\pi b|\sin \theta|+\epsilon) r]), \quad|\theta| \leqq \frac{1}{2} \pi$,

where $a \geqq 0, b \geqq-\frac{1}{2} a$, and $\epsilon$ is an arbitrary positive quantity. If $\left\{z_{n}\right\}$ is a sequence satisfying (1.2) and (1.3) and if

$$
D>b+\frac{1}{2} a \text {, }
$$

then

$$
\limsup _{n \rightarrow \infty} \frac{\log \left|\Phi\left(z_{n}\right)\right|}{\left|z_{n}\right|} \leqq \pi p
$$

implies

$$
\Phi\left(r e^{i \theta}\right)=O(\exp [\pi r(p \cos \theta+b|\sin \theta|+\epsilon)]) .
$$

Proof. We shall assume that $a>0$, for if $a=0$ we have Theorem II. Let

$$
\phi(z)=\frac{\Phi(z)}{\Gamma(1+a z)} .
$$

From Stirling's formula it follows easily that for $|\theta| \leqq \frac{1}{2} \pi$ and large $r$

$\log \left|\Gamma\left(1+a r e^{i \theta}\right)\right|-a r \log r \cos \theta+a r \theta \sin \theta+a r \cos \theta-\frac{1}{2} \log r=O(1)$.

Thus from (2.11)

(2.16) $\phi\left(r e^{i \theta}\right)=O(\exp [(\pi b|\sin \theta|+a \theta \sin \theta+a \cos \theta+\epsilon) r]), \quad|\theta| \leqq \frac{1}{2} \pi$, and from (2.13)

$$
\phi\left(z_{n}\right)=O\left(\exp \left[\left(-a \log \left|z_{n}\right| \cos \theta_{n}+\pi p+a+\epsilon\right)\left|z_{n}\right|\right]\right) .
$$

Using (2.12), (2.16), and (2.17) in Theorem II, we see that 


$$
\limsup _{r \rightarrow \infty} \frac{\log \left|\phi\left(r e^{i \theta}\right)\right|}{r}=-\infty, \quad|\theta|<\frac{1}{2} \pi .
$$

As in the proof of Theorem I we define

$$
g(z)=\sum_{1}^{\infty} \frac{\phi\left(z_{n}\right) e^{\Lambda z_{n}}}{F^{\prime}\left(z_{n}\right)\left(z-z_{n}\right)} e^{-A z} F(z),
$$

where here $A$ is any real number. We also consider $\psi(z)=\{\phi(z)-g(z)\} / F(z)$. As in Theorem $I, \psi(z)$ is an analytic function satisfying (2.8) for $|\mathrm{am} z| \leqq \frac{1}{2} \pi$. Along the imaginary axis (assuming $\Re z_{n}>1$ as we may with no restriction)

$$
|\psi(i y)| \leqq \max \left|\frac{\phi(i y)}{F(i y)}\right|+\sum_{1}^{\infty}\left|\frac{\phi\left(z_{n}\right) e^{A z_{n}}}{F^{\prime}\left(z_{n}\right)}\right| .
$$

By (2.1), (2.12), and (2.16), $|\phi(i y) / F(i y)|$ is bounded. Thus there exists some $M_{1}>0$, which is entirely independent of $A$, such that

$$
|\psi(i y)| \leqq M_{1}+\sum_{1}^{\infty}\left|\frac{\phi\left(z_{n}\right) e^{A z_{n}}}{F^{\prime}\left(z_{n}\right)}\right| .
$$

Along am $z=\frac{1}{4} \pi i$ we have, using (2.1), (2.18), and (2.19),

$$
\psi\left(r e^{\pi i / 4}\right)=O\left(\exp [-2 A r]+\exp \left[-A r \cos \frac{1}{4} \pi\right]\right) .
$$

Using this and (2.20) in Theorem A, we see that $\psi(z) e^{A z}$ is bounded in the sectors $\frac{1}{4} \pi \leqq \mathrm{am} z \leqq \frac{1}{2} \pi$ and $-\frac{1}{2} \pi \leqq \operatorname{am} z \leqq \frac{1}{4} \pi$, or in the entire right half-plane. Again from Theorem A, (2.20) now implies that

$$
\left|e^{A z} \psi(z)\right| \leqq M_{1}+\sum_{1}^{\infty}\left|\frac{\phi\left(z_{n}\right) e^{A z_{n}}}{F^{\prime}\left(z_{n}\right)}\right|, \quad \mid \text { am } z \mid \leqq \frac{1}{2} \pi .
$$

In particular then

$$
\left|\psi\left(r e^{\pi i / 4}\right)\right| \leqq \exp \left[-\operatorname{Ar} \cos \frac{1}{4} \pi\right]\left(M_{1}+\sum_{1}^{\infty}\left|\frac{\phi\left(z_{n}\right) e^{\Delta z_{n}}}{F^{\prime}\left(z_{n}\right)}\right|\right) .
$$

In the following we use $M_{2}, M_{3}$, etc., to represent positive constants independent of $A$. Using (2.19) and (2.1), we have

$$
\left|g\left(r e^{\pi i / 4}\right)\right| \leqq \exp \left[-\operatorname{Ar} \cos \frac{1}{4} \pi+\pi D r\right] M_{2} \sum_{1}^{\infty}\left|\frac{\phi\left(z_{n}\right) e^{A z_{n}}}{F^{\prime}\left(z_{n}\right)}\right| .
$$

Since $\phi(z)=g(z)+\psi(z) F(z)$ we have, using (2.1), (2.21), and (2.22),

$$
\left|\phi\left(r e^{\pi i / 4}\right)\right| \leqq \exp \left[-A r \cos \frac{1}{4} \pi+\pi D r\right] M_{3}\left(1+\sum_{1}^{\infty}\left|\frac{\phi\left(z_{n}\right) e^{A z_{n}}}{F^{\prime}\left(z_{n}\right)}\right|\right) .
$$


Or setting $A=a \log r$, we have

$$
\left|\phi\left(r e^{\pi i / 4}\right)\right|
$$

$$
\leqq \exp \left[-\operatorname{ar} \log r \cos \frac{1}{4} \pi+\pi D r\right] M_{3}\left(1+\sum_{1}^{\infty}\left|\frac{\phi\left(z_{n}\right) \exp \left[a z_{n} \log r\right]}{F^{\prime}\left(z_{n}\right)}\right|\right) .
$$

If we use (2.4) and (2.17), we have

$$
\begin{aligned}
\sum_{1}^{\infty}\left|\frac{\phi\left(z_{n}\right) \exp \left[a z_{n} \log r\right]}{F^{\prime}\left(z_{n}\right)}\right| \\
=O\left(\sum_{1}^{\infty} \exp \left[-a\left|z_{n}\right| \log \frac{\left|z_{n}\right|}{r} \cos \theta_{n}+(\pi p+a+\epsilon)\left|z_{n}\right|\right]\right) .
\end{aligned}
$$

But $\exp [-\lambda u \log (u / r)]$ has for its maximum value as $u$ varies $e^{\lambda r / e}$. Thus if $B>1$ is so large that $\log B>2(\pi p+a+\epsilon) / a$, then

$$
\begin{aligned}
\sum_{1}^{\infty}\left|\frac{\phi\left(z_{n}\right) \exp \left[a z_{n} \log r\right]}{F^{\prime}\left(z_{n}\right)}\right| & =O((r \exp [(\pi p+a+\epsilon) B r+a r / e] \\
& \left.+\sum_{\left|z_{n}\right|>B r} \exp \left[-\left|z_{n}\right|\left(2 \cos \theta_{n}-1\right)(\pi p+a+\epsilon)\right]\right) \\
& =O(\exp [B r(2 a+\pi p+\epsilon)]) .
\end{aligned}
$$

Using this in (2.23) gives

$$
\phi\left(r e^{\pi i / 4}\right)=O\left(\exp \left[-a r \log r \cos \frac{1}{4} \pi+C r\right]\right),
$$

where $C=\pi D+B(2 a+\pi p+\epsilon)$. Or in (2.15)

$$
\Phi\left(r e^{\pi i / 4}\right)=O\left(e^{C r}\right) .
$$

But this and (2.11) for $\theta= \pm \frac{1}{2} \pi$, used in Theorem $A$, show that $\Phi(z)$ is of exponential type in the right half-plane. Theorem III now follows at once from Theorem II.

THEOREM IV. If in Theorem III, (2.13) is replaced by

$$
\Phi\left(z_{n}\right)=O\left(\left|\exp \left[-k z_{n} \log \left|z_{n}\right|\right]\right|\right), \quad k>0,
$$

then (2.14) is replaced by

$$
\Phi\left(r e^{i \theta}\right)=O(\exp [(-k \log r \cos \theta+\pi b|\sin \theta|+\epsilon) r]), \quad|\theta| \leqq \frac{1}{2} \pi .
$$

Proof. This proof is identical with that of Theorem III except that in (2.17) we take account of (2.24) and replace $a$ by $a+k$. This modification will then cause a corresponding change in (2.23) where $a$ is again replaced by $a+k$ since now we set $A=(a+k) \log r$. This finally gives us 


$$
\Phi\left(r e^{\pi i / 4}\right)=O\left(\exp \left[-k r \log r \cos \frac{1}{4} \pi+C r\right]\right) .
$$

From this we see that $\Phi(z) \Gamma(1+k z)$ is of exponential type and therefore Theorem II can be applied to obtain Theorem IV.

THEOREM V. If $\Phi(z)$ satisfies the requirements of Theorem III with (2.13) replaced by (2.24) and

$$
k>2 b,
$$

then $\Phi(z)=0$.

In proving this and subsequent results of this type we use a fundamental theorem* of Carleman, or rather a consequence of this theorem.

Let $f(z)$ be analytic in the half-plane $|\mathrm{am} z| \leqq \frac{1}{2} \pi$ and let $R>1$. Then

$$
\begin{aligned}
\frac{1}{2 \pi} \int_{1}^{R}\left(\frac{1}{y^{2}}-\frac{1}{R^{2}}\right) \log \mid & f(i y) f(-i y) \mid d y \\
& +\frac{1}{\pi R} \int_{-\pi / 2}^{\pi / 2} \log \left|f\left(R e^{i \theta}\right)\right| \cos \theta d \theta+A>0,
\end{aligned}
$$

where $A$ is some number depending only on $f(z)$.

Proof. Applying (2.27) to $\Phi(z)$ we have, assuming it is not identically zero,

$$
\begin{aligned}
-A<\frac{1}{2 \pi} \int_{1}^{R}\left(\frac{1}{y^{2}}-\frac{1}{R^{2}}\right) \log ^{+}|\Phi(i y) \Phi(-i y)| d y & \\
& +\frac{1}{\pi R} \int_{-\pi / 2}^{\pi / 2} \log ^{+}\left|\Phi\left(r e^{i \theta}\right)\right| \cos \theta d \theta .
\end{aligned}
$$

Using (2.25) and replacing $A$ by another constant $A_{1}$, we get

$$
-A_{1}<(b+\epsilon) \log R-\frac{1}{2} k \log R,
$$

or $2(b+\epsilon) \geqq k$. But by $(2.26)$ this is impossible for arbitrarily small $\epsilon$. Thus $\Phi(z)=0$.

3. In this section we consider Theorem VI and related theorems. We recall that Theorem VI may be valid even when $D=L$. The proof is quite different from those of the previous section.

The method of this section can best be presented by first using it to give an alternative proof of Theorem II.

Alternative proof of Theorem II. There is no restriction in assuming that

$$
h\left(\frac{1}{2} \pi\right)=h\left(-\frac{1}{2} \pi\right)=\pi L .
$$

* E. C. Titchmarsh, The Theory of Functions, Oxford, 1932, p. 130. 
Moreover it is clear that (1.5) follows if we prove that

$$
\limsup _{n \rightarrow \infty} \frac{\log \left|\phi\left(z_{n}\right)\right|}{\left|z_{n}\right|} \leqq 0
$$

implies that $h(0) \leqq 0$.

As in (2.6)

$$
g(z)=\sum_{1}^{\infty} \frac{\phi\left(z_{n}\right) e^{-\epsilon z_{n}}}{F^{\prime}\left(z_{n}\right)\left(z-z_{n}\right)} e^{\epsilon z} F(z)
$$

for any $\epsilon>0$, is an entire function of exponential type. And as in (2.7)

$$
\psi(z)=\frac{\phi(z)-g(z)}{F(z)}
$$

is analytic for $|\mathrm{am} z| \leqq \frac{1}{2} \pi$. As in (2.8)

$$
\psi(z)=O(\exp [|z| \log |z|]), \quad \mid \text { am } z \mid \leqq \frac{1}{2} \pi .
$$

Since $\phi(z)$ and $g(z)$ are of exponential type in $|\mathrm{am} z| \leqq \frac{1}{2} \pi,(2.1)$ and (3.3) give

$$
\psi\left(r e^{\pi i / 4}\right)=O\left(e^{B r}\right)
$$

for some $B>0$. From (3.3) we have

$$
|\psi(i y)| \leqq\left|\frac{\phi(i y)}{F(i y)}\right|+\sum_{1}^{\infty}\left|\frac{\phi\left(z_{n}\right) e^{-\epsilon z_{n}}}{F^{\prime}\left(z_{n}\right)\left(i y-z_{n}\right)}\right| .
$$

Since $D>L,(3.0)$ and (2.1) imply that

$$
\phi(i y) / F(i y)=O\left(\exp \left[-\frac{1}{2} \pi(D-L)|y|\right]\right) .
$$

From (3.6) and (3.7)

$$
\psi(i y)=O\left(\frac{1}{|y|}\right) .
$$

Using (3.5) and (3.8), $z e^{-2 B z} \psi(z)$ is bounded along the imaginary axis and the line am $z=\frac{1}{2} \pi$. By Theorem A this and (3.4) implies that it is bounded in the entire right half-plane. Thus.

$$
\frac{\psi(z) e^{-2 B z}}{1+z}=O\left(\frac{1}{|z|^{2}}\right) .
$$

When we use this, it follows at once from the Cauchy integral theorem that for $x>0$, 
Or if

$$
\begin{aligned}
\frac{\psi(z) e^{-2 B z}}{1+z} & =\frac{1}{2 \pi i} \int_{-i \infty}^{i \infty} \frac{\psi(s) e^{-2 B s}}{1+s} \frac{d s}{z-s} \\
& =\frac{1}{2 \pi i} \int_{-i \infty}^{i \infty} \frac{\psi(s) e^{-2 B s}}{1+s} d s \int_{0}^{\infty} e^{-u(z-s)} d u \\
& =\frac{1}{2 \pi i} \int_{0}^{\infty} e^{-u z} d u \int_{-i \infty}^{i \infty} \frac{\psi(s) e^{-2 B s}}{1+s} e^{u s} d s
\end{aligned}
$$

$$
H(u)=\frac{1}{2 \pi i} \int_{-i \infty}^{i \infty} \frac{\psi(s) e^{-2 B s}}{1+s} e^{u s} d s
$$

then for $x>0$,

$$
\frac{\psi(z) e^{-2 B z}}{1+z}=\int_{0}^{\infty} H(u) e^{-u z} d u .
$$

When we use (3.9) and close the path of integration to the right in (3.10), it is clear that

$$
H(u)=0, \quad u<0 .
$$

On the other hand, when we use (3.3) in (3.10), it follows that

$$
\begin{aligned}
H(u)= & \frac{1}{2 \pi} \int_{-i \infty}^{i \infty} \frac{\phi(i t) e^{-2 i B t}}{F(i t)(1+i t)} e^{i u t} d t \\
& -\sum_{1}^{\infty} \frac{\phi\left(z_{n}\right) e^{-\epsilon z_{n}}}{F^{\prime}\left(z_{n}\right)} \frac{1}{2 \pi i} \int_{-i \infty}^{i \infty} \frac{e^{s(u-2 B+\epsilon)}}{(1+s)\left(s-z_{n}\right)} d s .
\end{aligned}
$$

Or for $u<2 B-\epsilon$,

$$
H(u)=\frac{1}{2 \pi} \int_{-\infty}^{\infty} \frac{\phi(i t) e^{-2 B i t}}{F(i t)(1+i t)} e^{i u t} d t-\sum_{1}^{\infty} \frac{\phi\left(z_{n}\right) e^{z_{n}(u-2 B)}}{F^{\prime}\left(z_{n}\right)\left(1+z_{n}\right)} .
$$

Clearly by (3.1), (2.4), and (1.2) the infinite series on the right of (3.14) represents an analytic function for $u<2 B$. Again by (3.7) it follows that the infinite integral on the right of (3.14) represents an analytic function in $u$ for $-\infty<u<\infty$. Since the sum of two analytic functions is analytic it follows that $H(u)$ is analytic for $u<2 B-\epsilon$. But by (3.12), $H(u)=0, u<0$. Therefore $H(u)=0, u<2 B-\epsilon$. Using this in (3.11), we have

$$
\frac{\psi(x) e^{-2 B x}}{1+x}=\int_{2 B-\epsilon}^{\infty} e^{-u x} H(u) d u .
$$


By (3.9) and (3.10), $H(u)$ is bounded. Thus

$$
\frac{\psi(x) e^{-2 B x}}{1+x}=O\left(e^{-x(2 B-\epsilon)}\right)
$$

or $\psi(x)=O\left(e^{2 e x}\right)$. If we recall that $\phi(x)=g(x)+F(x) \psi(x)$, it follows that

$$
h(0)=\limsup _{x \rightarrow \infty} \frac{\log |\phi(x)|}{x} \leqq 2 \epsilon .
$$

Since $\epsilon$ is arbitrary, $h(0) \leqq 0$. This completes the proof.

The difference between this proof and those of $\$ 2$ is that here we get a representation of $\psi(z)$ in terms of $H(u)$. In this section we are attempting to refine Theorem II so that $D>L,(1.7)$, is not necessary. Let us see how such a change would affect the argument in the preceding theorem. It is clear that the crucial point in this argument is the paragraph following (3.14) and it is only with this that we need concern ourselves here.

It is convenient to write (3.14) as

$$
H(u)=H_{1}(u)-H_{2}(u), \quad u<2 B-\epsilon,
$$

where

$$
H_{1}(u)=\frac{1}{2 \pi} \int_{-\infty}^{\infty} \frac{\phi(i t) e^{-2 i B t}}{F(i t)(1+i t)} e^{i u t} d t
$$

and

$$
H_{2}(u)=\sum_{1}^{\infty} \frac{\phi\left(z_{n}\right) e^{z_{n}(u-2 B)}}{F^{\prime}\left(z_{n}\right)\left(1+z_{n}\right)} .
$$

It is clear that $H_{2}(u)$ is analytic for $u<2 B$ irrespective of how $D$ compares with $L$. Therefore it is only with $H_{1}(u)$ that we need be concerned in changing (1.7). If $D=L$, then there need exist no $\delta>0$ such that

$$
\phi(i t) / F(i t)=O\left(e^{-\delta|t|}\right)
$$

and $H_{1}(u)$ need no longer be analytic.

Is there any weaker condition than analyticity on $H_{1}(u)$ that tells us that if $H_{1}(u)=H_{2}(u), u<0$, and $H_{2}(u)$ is analytic for $u<a$, then $H_{1}(u)=H_{2}(u)$, $u<a$ ? That there are such weaker conditions is shown by the following result.

THEOREM B. If

$$
f(u)=\int_{-\infty}^{\infty} G(t) e^{i t u} d t,
$$

where $G(t) \epsilon L(-\infty, \infty)$ and if for $t>0$ 


$$
G(t)=O\left(e^{-\theta(t)}\right),
$$

where $\theta(t)$ is a monotone non-decreasing function such that

$$
\int_{1}^{\infty} \frac{\theta(t)}{t^{2}} d t=\infty
$$

then if $f(u)$ coincides with an analytic function over some interval it coincides with the analytic function over its entire interval of analyticity on the $u$ axis.*

In order to use this theorem with $H_{1}(u)$ for $f(u)$ it must be shown that $\phi(i t) / F(i t)$ satisfies a condition of the type (3.19). under the hypothesis of Theorem VI. This is done by

LEMMA 2. If

$$
F(z)=\prod_{1}^{\infty}\left(1-\frac{z^{2}}{\lambda_{n}^{2}}\right)
$$

and if $\left\{\lambda_{n}\right\}$ satisfies the requirements of Theorem VI, then there exists a nondecreasing function $\theta(y), y>0$, such that for sufficiently large $|y|$

$$
e^{x L|y|} / F(i y)=O\left(|y|{ }^{2 C} e^{-\theta(|y|)}\right),
$$

and such that

$$
\int_{1}^{\infty} \frac{\theta(y)}{y^{2}} d y=\infty
$$

Proof. We assume that $\lambda_{1} \geqq 1$ (since we can discard any $\lambda_{n}$ 's which are less than one). Clearly

Since

$$
\begin{aligned}
\log |F(i y)| & =\int_{0}^{\infty} d \Lambda(u) \log \left(1+\frac{y^{2}}{u^{2}}\right) \\
& =2 \int_{0}^{\infty} \frac{\Lambda(u)}{u} \frac{y^{2}}{y^{2}+u^{2}} d u .
\end{aligned}
$$

we have

$$
2 \int_{0}^{\infty} \frac{y^{2}}{y^{2}+u^{2}} d u=\pi|y|
$$

* In On a class of non-vanishing functions, Proceedings of the London Mathematical Society, vol. 41 (1936), p. 393, it is shown that if $f(u)$ vanishes over any interval it vanishes identically. Theorem B is closely related to this result. The proof of Theorem B will appear shortly in the Journal for Mathematics and Physics. 


$$
\begin{aligned}
\log & |F(i y)|-\pi L|y|=2 \int_{0}^{\infty} \frac{\Lambda(u)-L u}{u} \frac{y^{2}}{y^{2}+u^{2}} d u \\
= & 4 \int_{0}^{\infty} d u \frac{y^{2} u}{\left(y^{2}+u^{2}\right)^{2}} \int_{0}^{\infty} \frac{\Lambda(u)-L u}{u} d u \\
= & 4 \int_{0}^{\infty} \frac{y^{2} u}{\left(y^{2}+u^{2}\right)^{2}} d u \int_{1}^{u}(\Lambda(v)-L v+C) \frac{d v}{v}-4 L \int_{0}^{\infty} \frac{y^{2} u}{\left(y^{2}+u^{2}\right)^{2}} d u \\
& \quad-4 C \int_{0}^{\infty} \frac{y^{2} u \log u}{\left(y^{2}+u^{2}\right)^{2}} d u \\
\geqq & 4 \int_{|v|}^{\infty} \frac{y^{2} u}{\left(y^{2}+u^{2}\right)^{2}} d u \int_{1}^{|y|}(\Lambda(v)-L v+C) \frac{d v}{v}-4(L+C)-2 C \log |y| \\
= & \int_{1}^{|y|} \frac{\Lambda(v)-L v+C}{v} d v-2 C \log |y|+\text { constant. }
\end{aligned}
$$

If we set

$$
\theta(y)=\int_{1}^{v} \frac{\Lambda(v)-L v+C}{v} d v, \quad y>1,
$$

then by (1.11), $\theta(y)$ is non-decreasing. Also

$$
\begin{aligned}
\int_{1}^{\infty} \frac{\theta(y)}{y^{2}} d y & =\int_{1}^{\infty} \frac{d y}{y^{2}} \int_{1}^{v} \frac{\Lambda(v)-L v+C}{v} d v \\
& =\int_{1}^{\infty} \frac{\Lambda(v)-L v+C}{v^{2}} d v=\infty .
\end{aligned}
$$

This proves the lemma.

Proof of Theorem VI. Here, as in the alternative proof of Theorem II, it suffices to show that $\lim \sup \log \left|\phi\left(\lambda_{n}\right)\right| / \lambda_{n} \leqq 0$ implies $h(0) \leqq 0$. The proof proceeds almost exactly like that of the alternative proof of Theorem II up to (3.14) except that we concern ourselves with $e^{-2 B} \psi(z) /(1+z)^{2 C+2}$ here. So in place of (3.14) we get

$$
\begin{aligned}
H(u)=\frac{1}{2 \pi} \int_{-i \infty}^{i \infty} \frac{\phi(i y) e^{-2 i B y}}{F(i y)(1+i y)^{2 C+2}} e^{i u y} d y-\sum_{1}^{\infty} \frac{\phi\left(\lambda_{n}\right) e^{\lambda_{n}(u-2 B)}}{F^{\prime}\left(\lambda_{n}\right)\left(1+\lambda_{n}\right)^{2 C+2}}, & \\
u & <2 B-\epsilon .
\end{aligned}
$$

As before the infinite series on the right is analytic for $u<2 B$ and $H(u)=0$, $u<0$, or by (3.15), $H_{1}(u)=H_{2}(u), u<0$. By Lemma 2

$$
H_{1}(u)=\frac{1}{2 \pi} \int_{-i \infty}^{i \infty} \frac{\phi(i y) e^{-2 i B y}}{F(i y)(1+i y)^{2 C+2}} e^{i u y} d y
$$


satisfies the requirements of Theorem B. Since

$$
H_{1}(u)=H_{2}(u)=\sum_{1}^{\infty} \frac{\phi\left(\lambda_{n}\right) e^{\lambda_{n}(u-2 B)}}{F^{\prime}\left(\lambda_{n}\right)\left(1+\lambda_{n}\right)^{2 C+2}}, \quad u<0,
$$

Theorem $\mathrm{B}$ implies that this is true for $u<2 B-\epsilon$. That is $H(u)=0$ for $u<2 B-\epsilon$. As in the alternative proof of Theorem II, this leads at once to $\psi(x)=O\left(e^{2 e x}\right) \cdot h(0) \leqq 0$ follows at once completing the proof.

There are variations of Theorem VI such as the following:

THEOREM VI-A. Theorem VI remains true if (1.10) is replaced by

$$
\phi(i y)=O(\exp [\pi L|y|-\theta(|y|)]),
$$

where $\theta(y), y>0$, is a non-decreasing function of $y$ which satisfies (3.20).

The proof of this result is quite obvious from what precedes.

Before proving Theorem VII the following result very much like Lemma 2 is necessary.

LEMMA 3. Let $F(z)$ be defined as in (2.0) with $\left\{z_{n}\right\}$ satisfying the requirements of Theorem VII. Then

$$
\frac{1}{F(i y)}=O\left(e^{-\theta(|y|)}\right)
$$

where $\theta(y), y>0$, is a non-decreasing function of $y$ satisfying (3.20).

If $z_{n}=r_{n} e^{i \theta_{n}}$, there is no loss of generality in assuming that $\left|\theta_{n}\right|<\frac{1}{4} \pi$. We then have, if $\Lambda(u)$ is the number of $\left|z_{n}\right|<u$,

$$
\begin{aligned}
\log |F(i y)| & =\frac{1}{2} \sum_{1}^{\infty} \log \left(1+\frac{2 y^{2}}{r_{n}^{2}} \cos 2 \theta_{n}+\frac{y^{4}}{r_{n}^{4}}\right) \\
& \geqq \frac{1}{2} \sum_{1}^{\infty} \log \left(1+\frac{y^{4}}{r_{n}^{4}}\right)=\frac{1}{2} \int_{0}^{\infty} d \Lambda(u) \log \left(1+\frac{y^{4}}{u^{4}}\right) \\
& >\frac{\log 2}{2} \int_{0}^{|y|} d \Lambda(u)>\frac{1}{4} \Lambda(|y|) .
\end{aligned}
$$

If we take $\theta(y)=\Lambda(|y|)$, then $|F(i y)|^{-1}=O\left(\exp \left[-\frac{1}{4} \theta(|y|)\right]\right)$. To prove (3.20) we have

$$
\int_{0}^{\infty} \frac{\Lambda(y)}{y^{2}} d y=\lim _{A \rightarrow \infty}\left[\int_{0}^{A} \frac{d \Lambda(y)}{y}-\frac{\Lambda(A)}{A}\right]
$$


By (1.8)

$$
\int_{0}^{\infty} \frac{d \Lambda(y)}{y}=\infty
$$

and therefore (3.20) is satisfied.

Proof of Theorem VII. To show that (1.8) is sufficient we proceed exactly, as in the alternative proof of Theorem II up to (3.14). We then use Lemma 3 to apply Theorem $\mathrm{B}$ to $H_{1}(u),(3.16)$, and show that $H(u)=0, u<2 B-\epsilon$. From this the fact that (3.1) implies $\dot{n}(0) \leqq 0$ follows at once.

We now turn to the necessity of condition (1.8). Let us assume that

$$
\sum_{1}^{\infty} \frac{1}{\left|z_{n}\right|}<\infty \text {. }
$$

Then we shall show that no such result as (1.5) holds. Let

$$
\phi(z)=\prod_{1}^{\infty} \frac{\left(z-z_{n}\right)\left(z-\bar{z}_{n}\right)}{\left(z+z_{n}\right)\left(z+\bar{z}_{n}\right)} .
$$

Then $\phi(z)$ is analytic in the right half-plane. Moreover

$$
\left|\phi\left(r e^{i \theta}\right)\right|=\prod_{1}^{\infty}\left|\frac{r e^{i \theta}-z_{n}}{r e^{i \theta}+z_{n}}\right|\left|\frac{r e^{i \theta}-\bar{z}_{n}}{r e^{i \theta}+\bar{z}_{n}}\right| \leqq 1, \quad|\theta| \leqq \frac{1}{2} \pi .
$$

Therefore $\phi(z)$ satisfies the requirements of Theorem VII. Moreover

$$
\limsup _{n \rightarrow \infty} \frac{\log \left|\phi\left(z_{n}\right)\right|}{\left|z_{n}\right|}=-\infty .
$$

If (1.5) could be applied it would give

$$
\limsup _{x \rightarrow \infty} \frac{\log |\phi(x)|}{x}=-\infty .
$$

Applying Theorem A to $e^{C z} \phi(z), C>0$, in the upper and lower right quadrants we see that it is bounded. Then again applying Theorem $A$ to the bounded function $e^{C_{z}} \phi(z)$ in the right half-plane, we see that for $|\operatorname{am} z| \leqq \frac{1}{2} \pi$, $\left|e^{C_{z}} \phi(z)\right| \leqq 1$. Since $C$ can be made arbitrarily large this means that $\phi(z)=0$, which obviously is not the case. Thus (1.8) is a necessary condition in order that Theorem VII be true.

The analogue of Theorem III in this section is the following:

ThEOREM VIII. Let $\Phi(z)$ be an analytic function in the right half-plane $|\mathrm{am} z| \leqq \frac{1}{2} \pi$ such that for any $\epsilon>0$

$$
\Phi\left(r e^{i \theta}\right)=O(\exp [(a \log r \cos \theta+\epsilon \cos \theta+b|\cdot \sin \theta|) r]), \quad|\theta| \leqq \frac{1}{2} \pi,
$$


where $a \geqq 0, b \geqq-\frac{1}{2} a$, and let $\left\{\lambda_{n}\right\}$ be a positive increasing sequence satisfying (1.9). Let $\Lambda(u)$ be the number of $\lambda_{n}<u$. If

$$
\int_{1}^{\infty} \frac{\Lambda(u)-\left(b+\frac{1}{2} a\right) u}{u^{2}} d u=\infty
$$

if for some $C, \Lambda(u)>\left(b+\frac{1}{2} a\right) u-C$, and

$$
\limsup _{n \rightarrow \infty} \frac{\log \left|\Phi\left(\lambda_{n}\right)\right|}{\lambda_{n}} \leqq \pi p,
$$

then

$$
\Phi\left(r e^{i \theta}\right)=O(\exp [\pi r(p \cos \theta+b|\sin \theta|+\epsilon \cos \theta)]), \quad|\theta| \leqq \frac{1}{2} \pi,
$$

where $\epsilon$ is an arbitrary positive quantity.

Proof. As in Theorem III we consider $\phi(z)=\Phi(z) / \Gamma(1+a z)$. By Theorem VI it follows that (2.18) holds. $g(z)$ is defined as in (2.19) and $\psi(z)$ $=\{\phi(z)-g(z)\} / F(z)$.

In formulas analogous to (2.20), (2.21), and so on, we consider $\psi(z) /(1+z)^{2 C}$ rather than just $\psi(z)$ as in Theorem III. Otherwise the proof now proceeds in precisely the same way as in Theorem III.

TheOREM IX. If in Theorem VIII (3.24) is replaced by

$$
\Phi\left(\lambda_{n}\right)=O\left(\exp \left[-k \lambda_{n} \log \left|\lambda_{n}\right|\right]\right), \quad k>0,
$$

then (3.25) is replaced by

$$
\Phi\left(r e^{i \theta}\right)=O(\exp [(-k \log r \cos \theta+\epsilon \cos \theta+\pi b|\sin \theta|) r]) .
$$

Proof. This theorem is related to Theorem VIII in the same way as Theorem IV is related to Theorem III and its proof follows almost at once from that of Theorem VIII just as that of Theorem IV follows almost at once from Theorem III.

THEOREM X. Let $\Phi(z)$ satisfy the requirements of Theorem VIII with (3.24) replaced by

$$
\limsup _{n \rightarrow \infty} \frac{\log \left|\Phi\left(\lambda_{n}\right)\right|+2 b \lambda_{n} \log \lambda_{n}}{\lambda_{n}}=-\infty .
$$

Then $\Phi(z)=0$.

Proof. Applying Theorem IX to $e^{B z} \Phi(z)$ for any $B>0$, we have

$$
\begin{gathered}
|\Phi(z)| \leqq B_{1}(\exp [(-2 b \log r \cos \theta-B \cos \theta+\pi b|\sin \theta|) r]), \\
|\theta| \leqq \frac{1}{2} \pi,
\end{gathered}
$$


where $B_{1}$ is a constant depending on $B$. Applying (2.27) to $\Phi(z)$ and using (3.27) we have for some $A_{1}$ depending only on $\Phi(z)$ (if $\Phi(z)$ does not vanish identically),

$$
-A_{1} \leqq \frac{1}{2 \pi} \int_{1}^{R}\left(\frac{1}{y^{2}}-\frac{1}{R^{2}}\right)(2 \pi b y) d y+\frac{1}{\pi R} \int_{-\pi / 2}^{\pi / 2} \log ^{+}\left|\Phi\left(R e^{i \theta}\right)\right| \cos \theta d \theta .
$$

Again using (3.27)

$-A_{1}-b \log R \leqq-\frac{2 b \log R+B}{\pi} \int_{-\pi / 2}^{\pi / 2} \cos ^{2} \theta d \theta+b \int_{-\pi / 2}^{\pi / 2} \cos \theta|\sin \theta| d \theta$

$$
+\frac{\log B_{1}}{\pi R} \int_{-\pi / 2}^{\pi / 2} \cos \theta d \theta \text {. }
$$

Or

$$
-A_{1} \leqq-\frac{1}{2} B+b+\frac{2}{\pi R} \log B_{1} .
$$

Letting $R \rightarrow \infty$ we see that by choosing $B>2 A_{1}+2 b$ we obtain a contradiction.

Princeton University

AND

INSTITUTE fOR Advanced Study,

Prnnceton, N. J. 